\title{
Opposite Antipodal Fundamental Solution of Laplace's Equation in Hyperspherical Geometry
}

\author{
Howard S. COHL †‡ \\ $\dagger$ Applied and Computational Mathematics Division, Information Technology Laboratory, \\ National Institute of Standards and Technology, Mission Viejo, California, 92694 USA \\ E-mail: howard.cohl@nist.gov \\ ¥Department of Mathematics, University of Auckland, 38 Princes Str., Auckland, New Zealand
}

Received August 18, 2011, in final form November 22, 2011; Published online November 29, 2011

https://doi.org/10.3842/SIGMA.2011.108

\begin{abstract}
Due to the isotropy of $d$-dimensional hyperspherical space, one expects there to exist a spherically symmetric opposite antipodal fundamental solution for its corresponding Laplace-Beltrami operator. The $R$-radius hypersphere $\mathbf{S}_{R}^{d}$ with $R>0$, represents a Riemannian manifold with positive-constant sectional curvature. We obtain a spherically symmetric opposite antipodal fundamental solution of Laplace's equation on this manifold in terms of its geodesic radius. We give several matching expressions for this fundamental solution including a definite integral over reciprocal powers of the trigonometric sine, finite summation expressions over trigonometric functions, Gauss hypergeometric functions, and in terms of the Ferrers function of the second with degree and order given by $d / 2-1$ and $1-d / 2$ respectively, with argument $x \in(-1,1)$.
\end{abstract}

Key words: hyperspherical geometry; opposite antipodal fundamental solution; Laplace's equation; separation of variables; Ferrers functions

2010 Mathematics Subject Classification: 35A08; 35J05; 32Q10; 31C12; 33C05

\section{Introduction}

We compute closed-form expressions of a spherically symmetric opposite antipodal Green's function (opposite antipodal fundamental solution of Laplace's equation) for a $d$-dimensional Riemannian manifold of positive-constant sectional curvature, namely the $R$-radius hypersphere with $R>0$. This problem is intimately related to the solution of the Poisson equation on this manifold and the study of spherical harmonics which play an important role in exploring collective motion of many-particle systems in quantum mechanics, particularly nuclei, atoms and molecules. In these systems, the hyperradius is constructed from appropriately mass-weighted quadratic forms from the Cartesian coordinates of the particles. One then seeks either to identify discrete forms of motion which occur primarily in the hyperradial coordinate, or alternatively to construct complete basis sets on the hypersphere. This representation was introduced in quantum mechanics by Zernike \& Brinkman [39], and later invoked to greater effect in nuclear and atomic physics, respectively, by Delves [6] and Smith [33]. The relevance of this representation to two-electron excited states of the helium atom was noted by Cooper, Fano \& Prats [5]; Fock $[11,12]$ had previously shown that the hyperspherical representation was particularly efficient in representing the helium wave function in the vicinity of small hyperradii. There has been a rich literature of applications ever since. Examples include Zhukov [40] (nuclear structure), Fano [10] and Lin [26] (atomic structure), and Pack \& Parker [30] (molecular collisions). A recent monograph by Berakdar [2] discusses hyperspherical harmonic methods in the general context of highly-excited electronic systems. Useful background material relevant for the mathematical aspects of this paper can be found in [24, 35, 37]. Some historical references on this topic include [19, 25, 31, 32, 38]. 
This paper is organized as follows. In Section 2, we describe hyperspherical geometry and its corresponding metric, global geodesic distance function, Laplace-Beltrami operator (Laplacian), and hyperspherical global geodesic polar coordinate systems which parametrize points on this manifold. In Section 3, for hyperspherical geometry, we show how to compute 'radial' harmonics in a geodesic polar coordinate system and derive several alternative expressions for a 'radial' opposite antipodal fundamental solution of the Laplace's equation on the $R$-radius hypersphere.

Throughout this paper we rely on the following definitions. For $a_{1}, a_{2}, \ldots \in \mathbf{C}$, if $i, j \in \mathbf{Z}$ and $j<i$ then $\sum_{n=i}^{j} a_{n}=0$ and $\prod_{n=i}^{j} a_{n}=1$. The set of natural numbers is given by $\mathbf{N}:=\{1,2,3, \ldots\}$, the set $\mathbf{N}_{0}:=\{0,1,2, \ldots\}=\mathbf{N} \cup\{0\}$, the set of integers is given by $\mathbf{Z}:=\{0, \pm 1, \pm 2, \ldots\}$, the sets of real and complex numbers are given by $\mathbf{R}$ and $\mathbf{C}$ respectively.

\section{Hyperspherical geometry}

The Euclidean inner product for $\mathbf{R}^{d+1}$ is given by $(\mathbf{x}, \mathbf{y})=x_{0} y_{0}+x_{1} y_{1}+\cdots+x_{d} y_{d}$. The variety $(\mathbf{x}, \mathbf{x})=x_{0}^{2}+x_{1}^{2}+\cdots+x_{d}^{2}=R^{2}$, for $\mathbf{x} \in \mathbf{R}^{d+1}$ and $R>0$, defines the $R$-radius hypersphere $\mathbf{S}_{R}^{d}$. We denote the unit radius hypersphere by $\mathbf{S}^{d}:=\mathbf{S}_{1}^{d}$. Hyperspherical space in $d$-dimensions, denoted by $\mathbf{S}_{R}^{d}$, is a maximally symmetric, simply connected, $d$-dimensional Riemannian manifold with positive-constant sectional curvature (given by $1 / R^{2}$, see for instance [24, p. 148]), whereas Euclidean space $\mathbf{R}^{d}$ equipped with the Pythagorean norm, is a Riemannian manifold with zero sectional curvature.

Points on the $d$-dimensional hypersphere $\mathbf{S}_{R}^{d}$ can be parametrized using subgroup-type coordinate systems, i.e., those which correspond to a maximal subgroup chain $\mathrm{O}(d) \supset \cdots$ (see for instance [20, 22]). The isometry group of the space $\mathbf{S}_{R}^{d}$ is the orthogonal group $\mathrm{O}(d)$. Hyperspherical space $\mathbf{S}_{R}^{d}$, can be identified with the quotient space $\mathrm{O}(d) / \mathrm{O}(d-1)$. The isometry group $\mathrm{O}(d)$ acts transitively on $\mathbf{S}_{R}^{d}$. There exist separable coordinate systems on the hypersphere, analogous to parabolic coordinates in Euclidean space, which can not be constructed using maximal subgroup chains. Polyspherical coordinates, are coordinates which correspond to the maximal subgroup chain given by $\mathrm{O}(d) \supset \cdots$. What we will refer to as standard hyperspherical coordinates, correspond to the subgroup chain given by $\mathrm{O}(d) \supset \mathrm{O}(d-1) \supset \cdots \supset \mathrm{O}(2)$. (For a thorough discussion of polyspherical coordinates see [37, Section IX.5].) Polyspherical coordinates on $\mathbf{S}_{R}^{d}$ all share the property that they are described by $(d+1)$-variables: $R \in[0, \infty)$ plus $d$-angles each being given by the values $[0,2 \pi),[0, \pi],[-\pi / 2, \pi / 2]$ or $[0, \pi / 2]($ see $[20,21])$.

In our context, a useful subset of polyspherical coordinate are geodesic polar coordinates $(\theta, \widehat{\mathbf{x}})$ (see for instance [29]). These coordinates, which parametrize points on $\mathbf{S}_{R}^{d}$, have origin at $O=(R, 0, \ldots, 0) \in \mathbf{R}^{d+1}$ and are given by a 'radial' parameter $\theta \in[0, \pi]$ which parametrizes points along a geodesic curve emanating from $O$ in a direction $\widehat{\mathbf{x}} \in \mathbf{S}^{d-1}$. Geodesic polar coordinate systems partition $\mathbf{S}_{R}^{d}$ into a family of $(d-1)$-dimensional hyperspheres, each with a 'radius' $\theta:=\theta_{d} \in(0, \pi)$, on which all possible hyperspherical coordinate systems for $\mathbf{S}^{d-1}$ may be used (see for instance [37]). One then must also consider the limiting case for $\theta=0, \pi$ to fill out all of $\mathbf{S}_{R}^{d}$. Standard hyperspherical coordinates (see $[23,27]$ ) are an example of geodesic polar coordinates, and are given by

$$
\begin{aligned}
& x_{0}=R \cos \theta, \\
& x_{1}=R \sin \theta \cos \theta_{d-1}, \\
& x_{2}=R \sin \theta \sin \theta_{d-1} \cos \theta_{d-2}, \\
& \cdots \cdots \cdots \cdots \cdots \cdots \cdots \cdots \cdots \cdot \cos \theta_{2}, \\
& x_{d-2}=R \sin \theta \sin \theta_{d-1} \cdots \sin \theta_{2} \cos \phi, \\
& x_{d-1}=R \sin \theta \sin \theta_{d-1} \cdots \sin \theta_{2} \sin \phi, \\
& x_{d}=R \sin \theta \sin \theta_{d-1} \cdots \log
\end{aligned}
$$

$\theta_{i} \in[0, \pi]$ for $i \in\{2, \ldots, d\}, \theta=\theta_{d}$, and $\phi \in[0,2 \pi)$. 
In order to study an opposite antipodal fundamental solution of Laplace's equation on the hypersphere, we need to describe how one computes the geodesic distance in this space. Geodesic distances on $\mathbf{S}_{R}^{d}$ are simply given by arc lengths, angles between two arbitrary vectors, from the origin in the ambient Euclidean space (see for instance [24, p. 82]). Any parametrization of the hypersphere $\mathbf{S}_{R}^{d}$, must have $(\mathbf{x}, \mathbf{x})=x_{0}^{2}+\cdots+x_{d}^{2}=R^{2}$, with $R>0$. The distance between two points $\mathbf{x}, \mathbf{x}^{\prime} \in \mathbf{S}_{R}^{d}$ on the hypersphere is given by

$$
d\left(\mathbf{x}, \mathbf{x}^{\prime}\right)=R \gamma=R \cos ^{-1}\left(\frac{\left(\mathbf{x}, \mathbf{x}^{\prime}\right)}{(\mathbf{x}, \mathbf{x})\left(\mathbf{x}^{\prime}, \mathbf{x}^{\prime}\right)}\right)=R \cos ^{-1}\left(\frac{1}{R^{2}}\left(\mathbf{x}, \mathbf{x}^{\prime}\right)\right) .
$$

This is evident from the fact that the geodesics on $\mathbf{S}_{R}^{d}$ are great circles, i.e., intersections of $\mathbf{S}_{R}^{d}$ with planes through the origin of the ambient Euclidean space, with constant speed parametrizations.

In any geodesic polar coordinate system, the geodesic distance between two points on the submanifold is given by

$$
d\left(\mathbf{x}, \mathbf{x}^{\prime}\right)=R \cos ^{-1}\left(\frac{1}{R^{2}}\left(\mathbf{x}, \mathbf{x}^{\prime}\right)\right)=R \cos ^{-1}\left(\cos \theta \cos \theta^{\prime}+\sin \theta \sin \theta^{\prime} \cos \gamma\right),
$$

where $\gamma$ is the unique separation angle given in each polyspherical coordinate system used to parametrize points on $\mathbf{S}^{d-1}$. For instance, the separation angle $\gamma$ in standard hyperspherical coordinates is given through

$$
\cos \gamma=\cos \left(\phi-\phi^{\prime}\right) \prod_{i=1}^{d-2} \sin \theta_{i} \sin \theta_{i}{ }^{\prime}+\sum_{i=1}^{d-2} \cos \theta_{i} \cos \theta_{i}{ }^{\prime} \prod_{j=1}^{i-1} \sin \theta_{j} \sin \theta_{j}{ }^{\prime}
$$

Corresponding separation angle formulae for any hyperspherical coordinate system used to parametrize points on $\mathbf{S}^{d-1}$ can be computed using (2) and the associated formulae for the appropriate inner-products.

One can also compute the Riemannian (volume) measure $d \operatorname{vol}_{g}$ (see for instance $[18$, Section 3.4]), invariant under the isometry group $\mathrm{SO}(d)$, of the Riemannian manifold $\mathbf{S}_{R}^{d}$. For instance, in standard hyperspherical coordinates (1) on $\mathbf{S}_{R}^{d}$ the volume measure is given by

$$
\mathrm{dvol}_{g}=R^{d} \sin ^{d-1} \theta \mathrm{d} \theta \mathrm{d} \omega:=R^{d} \sin ^{d-1} \theta \mathrm{d} \theta \sin ^{d-2} \theta_{d-1} \cdots \sin \theta_{2} \mathrm{~d} \theta_{1} \cdots \mathrm{d} \theta_{d-1} .
$$

The distance $r \in[0, \infty)$ along a geodesic, measured from the origin, is given by $r=\theta R$. To show that the above volume measure (5) reduces to the Euclidean volume measure at small distances (see for instance [23]), we examine the limit of zero curvature, the flat-space limit. In order to do this, we take the limit $\theta \rightarrow 0^{+}$and $R \rightarrow \infty$ of the volume measure (5) which produces

$$
\operatorname{dvol}_{g} \sim R^{d-1} \sin ^{d-1}\left(\frac{r}{R}\right) \mathrm{d} r \mathrm{~d} \omega \sim r^{d-1} \mathrm{~d} r \mathrm{~d} \omega,
$$

which is the Euclidean measure on $\mathbf{R}^{d}$, expressed in standard Euclidean hyperspherical coordinates. This measure is invariant under the Euclidean motion group $\mathrm{E}(d)$.

It will be useful below to express the Dirac delta distribution on $\mathbf{S}_{R}^{d}$. The Dirac delta distribution on the Riemannian manifold $\mathbf{S}_{R}^{d}$ with metric $g$ is defined for an open set $U \subset \mathbf{S}_{R}^{d}$ with $\mathbf{x}, \mathbf{x}^{\prime} \in \mathbf{S}_{R}^{d}$ such that

$$
\int_{U} \delta_{g}\left(\mathbf{x}, \mathbf{x}^{\prime}\right) \operatorname{dvol}_{g}= \begin{cases}1 & \text { if } \mathbf{x}^{\prime} \in U, \\ 0 & \text { if } \mathbf{x}^{\prime} \notin U .\end{cases}
$$

For instance, using (5) and (6), in standard hyperspherical coordinates on $\mathbf{S}_{R}^{d}$ (1), we see that the Dirac delta distribution is given by

$$
\delta_{g}\left(\mathbf{x}, \mathbf{x}^{\prime}\right)=\frac{\delta\left(\theta-\theta^{\prime}\right)}{R^{d} \sin ^{d-1} \theta^{\prime}} \frac{\delta\left(\theta_{1}-\theta_{1}^{\prime}\right) \cdots \delta\left(\theta_{d-1}-\theta_{d-1}^{\prime}\right)}{\sin \theta_{2}^{\prime} \cdots \sin ^{d-2} \theta_{d-1}^{\prime}} .
$$




\subsection{The Laplace and Poisson equations on the hypersphere}

Parametrizations of a submanifold embedded in Euclidean space can be given in terms of coordinate systems whose coordinates are curvilinear. These are coordinates based on some transformation that converts the standard Cartesian coordinates in the ambient space to a coordinate system with the same number of coordinates as the dimension of the submanifold in which the coordinate lines are curved.

The Laplace-Beltrami operator (Laplacian) in curvilinear coordinates $\boldsymbol{\xi}=\left(\xi^{1}, \ldots, \xi^{d}\right)$ on a Riemannian manifold is given by

$$
\Delta=\sum_{i, j=1}^{d} \frac{1}{\sqrt{|g|}} \frac{\partial}{\partial \xi^{i}}\left(\sqrt{|g|} g^{i j} \frac{\partial}{\partial \xi^{j}}\right),
$$

where $|g|=\left|\operatorname{det}\left(g_{i j}\right)\right|$, the metric is given by

$$
\mathrm{d} s^{2}=\sum_{i, j=1}^{d} g_{i j} \mathrm{~d} \xi^{i} \mathrm{~d} \xi^{j},
$$

and

$$
\sum_{i=1}^{d} g_{k i} g^{i j}=\delta_{k}^{j}
$$

where $\delta_{i}^{j} \in\{0,1\}$ is the Kronecker delta

$$
\delta_{i}^{j}:= \begin{cases}1 & \text { if } i=j, \\ 0 & \text { if } i \neq j,\end{cases}
$$

for $i, j \in \mathbf{Z}$. The relationship between the metric tensor $G_{i j}=\operatorname{diag}(1, \ldots, 1)$ in the ambient space and $g_{i j}$ of (7) and (8) is given by

$$
g_{i j}(\xi)=\sum_{k, l=0}^{d} G_{k l} \frac{\partial x^{k}}{\partial \xi^{i}} \frac{\partial x^{l}}{\partial \xi^{j}} .
$$

The Riemannian metric in a geodesic polar coordinate system on the submanifold $\mathbf{S}_{R}^{d}$ is given by

$$
\mathrm{d} s^{2}=R^{2}\left(\mathrm{~d} \theta^{2}+\sin ^{2} \theta \mathrm{d} \gamma^{2}\right),
$$

where an appropriate expression for $\gamma$ in a curvilinear coordinate system is given. If one combines (1), (4), (7) and (10), then in a geodesic polar coordinate system, Laplace's equation on $\mathbf{S}_{R}^{d}$ is given by

$$
\Delta f=\frac{1}{R^{2}}\left[\frac{\partial^{2} f}{\partial \theta^{2}}+(d-1) \cot \theta \frac{\partial f}{\partial \theta}+\frac{1}{\sin ^{2} \theta} \Delta_{\mathbf{S}^{d-1}} f\right]=0,
$$

where $\Delta_{\mathbf{S}^{d-1}}$ is the corresponding Laplace-Beltrami operator on $\mathbf{S}^{d-1}$.

Consider Poisson's equation on $\mathbf{S}_{R}^{d},-\Delta u=\rho$ on a compact Riemannian manifold $M$ with boundary $\partial M$. The divergence theorem on this manifold is given by (cf. [24, p. 43])

$$
\int_{M} \operatorname{div} \mathbf{X} \mathrm{d} V=\int_{\partial M}\langle\mathbf{X}, \mathbf{N}\rangle \mathrm{d} \tilde{V}
$$


where $\mathrm{d} V$ is the Riemannian volume measure on $M, \mathbf{N}$ is the outward unit normal to $\partial M$, and $\mathrm{d} \tilde{V}$ is the Riemannian volume measure of the induced metric on $\partial M$. If one invokes the divergence theorem on $\mathbf{S}_{R}^{d}$ with regard to Poisson's equation on this manifold using $\mathbf{X}=\nabla u$, then since $\partial \mathbf{S}_{R}^{d}=\varnothing$, one ascertains

$$
\int_{\mathbf{S}_{R}^{d}} \rho \mathrm{d} V=0
$$

Hence on $\mathbf{S}_{R}^{d}$ (and on all compact manifolds without boundary), there does not exist a source density distribution $\rho$, satisfying Poisson's equation, with non-vanishing integral. In fact, a fundamental solution of Laplace's equation on $\mathbf{S}_{R}^{d}$ (see Theorem 1 below), which has been pointed out in [4, Section 5.4], is actually the solution to the Poisson equation whose inhomogeneous source distribution is given by a point source at the origin and another with opposite sign, on the opposite pole of the hypersphere (both modeled by Dirac delta distributions).

We define the opposite antipodal fundamental solution of Laplace's equation $\mathcal{A}_{R}^{d}\left(\mathbf{x}, \mathbf{x}^{\prime}\right)$, as the solution to the following distributional partial differential equation

$$
-\Delta \mathcal{A}_{R}^{d}\left(\mathbf{x}, \mathbf{x}^{\prime}\right)=\delta_{g}\left(\mathbf{x}, \mathbf{x}^{\prime}\right)-\delta_{g}\left(-\mathbf{x}, \mathbf{x}^{\prime}\right),
$$

where $g$ is the Riemannian metric on $\mathbf{S}_{R}^{d}$ (e.g., (10)) and $\delta_{g}$ is the Dirac delta distribution on the manifold $\mathbf{S}_{R}^{d}$. The total integral over the entire manifold of this source distribution vanishes. Therefore the solution to the resulting partial differential equation must exist. The opposite antipodal fundamental solution is the most natural fundamental solution of Laplace's equation on a $d$-dimensional $R$-radius hypersphere $\mathbf{S}_{R}^{d}$ because (1) it is spherically symmetric and (2) its density distribution is composed wholly of the minimum number, two, of isolated Dirac delta distributions. Note that many other composed density distribution may be assembled by collecting a finite number of Dirac delta distributions, or by assembling an infinite number of Dirac delta distributions over the manifold.

\section{An opposite antipodal Green's function on the hypersphere}

\subsection{Harmonics in geodesic polar coordinates}

The harmonics (solutions to Laplace's equation) in a geodesic polar coordinate system are given in terms of a 'radial' solution ('radial' harmonics) multiplied by the angular solution (angular harmonics).

Using polyspherical coordinates on $\mathbf{S}^{d-1}$, one can compute the normalized hyperspherical harmonics in this space by solving the Laplace equation using separation of variables. This results in a general procedure which, for instance, is given explicitly in [20, 21]. These angular harmonics are given as general expressions involving trigonometric functions, Gegenbauer polynomials and Jacobi polynomials. The angular harmonics are eigenfunctions of the LaplaceBeltrami operator on $\mathbf{S}^{d-1}$ which satisfy the following eigenvalue problem (see for instance [34, equation (12.4) and Corollary 2 to Theorem 10.5])

$$
\Delta_{\mathbf{S}^{d-1}} Y_{l}^{K}(\widehat{\mathbf{x}})=-l(l+d-2) Y_{l}^{K}(\widehat{\mathbf{x}}),
$$

where $\widehat{\mathbf{x}} \in \mathbf{S}^{d-1}, Y_{l}^{K}(\widehat{\mathbf{x}})$ are normalized angular hyperspherical harmonics, $l \in \mathbf{N}_{0}$ is the angular momentum quantum number, and $K$ stands for the set of $(d-2)$-quantum numbers identifying degenerate harmonics for each $l$ and $d$. The degeneracy

$$
(2 l+d-2) \frac{(d-3+l) !}{l !(d-2) !}
$$


(see [37, equation (9.2.11)]), tells you how many linearly independent solutions exist for a particular $l$ value and dimension $d$. The angular hyperspherical harmonics are normalized such that

$$
\int_{\mathbf{S}^{d-1}} Y_{l}^{K}(\widehat{\mathbf{x}}) \overline{Y_{l^{\prime}}^{K^{\prime}}(\widehat{\mathbf{x}})} \mathrm{d} \omega=\delta_{l}^{l^{\prime}} \delta_{K}^{K^{\prime}}
$$

where $\mathrm{d} \omega$ is the Riemannian (volume) measure on $\mathbf{S}^{d-1}$, which is invariant under the isometry group $\mathrm{SO}(d)$ (cf. (5)), and for $x+\mathrm{i} y=z \in \mathbf{C}, \bar{z}=x-\mathrm{i} y$, represents complex conjugation. The angular solutions (hyperspherical harmonics) are well-known (see [37, Chapter IX] and [9, Chapter 11]). The generalized Kronecker delta symbol $\delta_{K}^{K^{\prime}}$ (cf. (9)) is defined such that it equals 1 if all of the $(d-2)$-quantum numbers identifying degenerate harmonics for each $l$ and $d$ coincide, and equals zero otherwise.

We now focus on the 'radial' solutions (harmonics) on $\mathbf{S}_{R}^{d}$. These satisfy the following ordinary differential equation (cf. (11) and (14))

$$
\frac{\mathrm{d}^{2} u}{\mathrm{~d} \theta^{2}}+(d-1) \cot \theta \frac{\mathrm{d} u}{\mathrm{~d} \theta}-\frac{l(l+d-2)}{\sin ^{2} \theta} u=0
$$

Four solutions of this ordinary differential equation $u_{1, \pm}^{d, l}, u_{2, \pm}^{d, l}:(-1,1) \rightarrow \mathbf{C}$ are given by

$$
u_{1, \pm}^{d, l}(\cos \theta):=\frac{1}{(\sin \theta)^{d / 2-1}} \mathrm{P}_{d / 2-1}^{ \pm(d / 2-1+l)}( \pm \cos \theta)
$$

and

$$
u_{2, \pm}^{d, l}(\cos \theta):=\frac{1}{(\sin \theta)^{d / 2-1}} Q_{d / 2-1}^{ \pm(d / 2-1+l)}( \pm \cos \theta)
$$

where $\mathrm{P}_{\nu}^{\mu}, \mathrm{Q}_{\nu}^{\mu}:(-1,1) \rightarrow \mathbf{C}$ are Ferrers functions of the first and second kind. The Ferrers functions of the first and second kind (see [28, Chapter 14]) can be defined respectively in terms of a sum over two Gauss hypergeometric functions, for all $\nu, \mu \in \mathbf{C}$ such that $\nu+\mu \notin-\mathbf{N}$,

$$
\begin{aligned}
\mathrm{P}_{\nu}^{\mu}(x):= & \frac{2^{\mu+1}}{\sqrt{\pi}} \sin \left[\frac{\pi}{2}(\nu+\mu)\right] \frac{\Gamma\left(\frac{\nu+\mu+2}{2}\right)}{\Gamma\left(\frac{\nu-\mu+1}{2}\right)} x\left(1-x^{2}\right)^{-\mu / 2}{ }_{2} F_{1}\left(\frac{1-\nu-\mu}{2}, \frac{\nu-\mu+2}{2} ; \frac{3}{2} ; x^{2}\right) \\
& +\frac{2^{\mu}}{\sqrt{\pi}} \cos \left[\frac{\pi}{2}(\nu+\mu)\right] \frac{\Gamma\left(\frac{\nu+\mu+1}{2}\right)}{\Gamma\left(\frac{\nu-\mu+2}{2}\right)}\left(1-x^{2}\right)^{-\mu / 2}{ }_{2} F_{1}\left(\frac{-\nu-\mu}{2}, \frac{\nu-\mu+1}{2} ; \frac{1}{2} ; x^{2}\right)
\end{aligned}
$$

(cf. [28, equation (14.3.11)]), and

$$
\begin{aligned}
& \mathrm{Q}_{\nu}^{\mu}(x):=\sqrt{\pi} 2^{\mu} \cos \left[\frac{\pi}{2}(\nu+\mu)\right] \frac{\Gamma\left(\frac{\nu+\mu+2}{2}\right)}{\Gamma\left(\frac{\nu-\mu+1}{2}\right)} x\left(1-x^{2}\right)^{-\mu / 2}{ }_{2} F_{1}\left(\frac{1-\nu-\mu}{2}, \frac{\nu-\mu+2}{2} ; \frac{3}{2} ; x^{2}\right) \\
& -\sqrt{\pi} 2^{\mu-1} \sin \left[\frac{\pi}{2}(\nu+\mu)\right] \frac{\Gamma\left(\frac{\nu+\mu+1}{2}\right)}{\Gamma\left(\frac{\nu-\mu+2}{2}\right)}\left(1-x^{2}\right)^{-\mu / 2}{ }_{2} F_{1}\left(\frac{-\nu-\mu}{2}, \frac{\nu-\mu+1}{2} ; \frac{1}{2} ; x^{2}\right)
\end{aligned}
$$

(cf. [28, equation (14.3.12)]). The Gauss hypergeometric function ${ }_{2} F_{1}: \mathbf{C} \times \mathbf{C} \times\left(\mathbf{C} \backslash-\mathbf{N}_{0}\right) \times$ $\mathbf{C} \backslash[1, \infty) \rightarrow \mathbf{C}$, can be defined in terms of the infinite series

$$
{ }_{2} F_{1}(a, b ; c ; z):=\sum_{n=0}^{\infty} \frac{(a)_{n}(b)_{n}}{(c)_{n} n !} z^{n}
$$


(see [28, equation (15.2.1)]), and elsewhere in $z$ by analytic continuation. On the unit circle $|z|=1$, the Gauss hypergeometric series converges absolutely if $\operatorname{Re}(c-a-b) \in(0, \infty)$, converges conditionally if $z \neq 1$ and $\operatorname{Re}(c-a-b) \in(-1,0]$, and diverges if $\operatorname{Re}(c-a-b) \in(-\infty,-1]$. For $z \in \mathbf{C}$ and $n \in \mathbf{N}_{0}$, the Pochhammer symbol $(z)_{n}$ (also referred to as the rising factorial) is defined as (cf. [28, equation (5.2.4)])

$$
(z)_{n}:=\prod_{i=1}^{n}(z+i-1) .
$$

The Pochhammer symbol is expressible in terms of a quotient of gamma functions as [28, equation (5.2.5)]

$$
(z)_{n}=\frac{\Gamma(z+n)}{\Gamma(z)}
$$

for all $z \in \mathbf{C} \backslash-\mathbf{N}_{0}$. The gamma function $\Gamma: \mathbf{C} \backslash-\mathbf{N}_{0} \rightarrow \mathbf{C}$ (see [28, Chapter 5]) is an important combinatoric function and is ubiquitous in special function theory. It is naturally defined over the right-half complex plane through Euler's integral (see [28, equation (5.2.1)])

$$
\Gamma(z):=\int_{0}^{\infty} t^{z-1} \mathrm{e}^{-t} \mathrm{~d} t
$$

Re $z>0$, and elsewhere by analytic continuation. The Euler reflection formula allows one to obtain values of the gamma function in the left-half complex plane [28, equation (5.5.3)], namely

$$
\Gamma(z) \Gamma(1-z)=\frac{\pi}{\sin \pi z}
$$

$0<\operatorname{Re} z<1$, for $\operatorname{Re} z=0, z \neq 0$, and then for $z$ shifted by integers using the following recurrence relation (see [28, equation (5.5.1)])

$$
\Gamma(z+1)=z \Gamma(z)
$$

An important formula which the gamma function satisfies is the duplication formula [28, equation (5.5.5)]

$$
\Gamma(2 z)=\frac{2^{2 z-1}}{\sqrt{\pi}} \Gamma(z) \Gamma\left(z+\frac{1}{2}\right)
$$

provided $2 z \notin-\mathbf{N}_{0}$.

Due to the fact that the space $\mathbf{S}_{R}^{d}$ is homogeneous with respect to its isometry group, the orthogonal group $\mathrm{O}(d)$, and therefore an isotropic manifold, we expect that there exist a fundamental solution on this space with spherically symmetric dependence. We specifically expect these solutions to be given in terms of the Ferrers function of the second kind with argument given by $\cos \theta$. The Ferrers function of the second kind naturally fits our requirements because it is singular at $\theta=0$, whereas the Ferrers function of the first kind, with the same argument, is regular at $\theta=0$. We require there to exist a singularity at the origin of an opposite antipodal fundamental solution of Laplace's equation on $\mathbf{S}_{R}^{d}$, since it is a manifold and must behave locally like a Euclidean fundamental solution of Laplace's equation which also has a singularity at the origin. 


\subsection{Opposite antipodal fundamental solution of $-\Delta u=0$ on $\mathrm{S}_{R}^{d}$}

In computing an opposite antipodal fundamental solution of the Laplacian on $\mathbf{S}_{R}^{d}$, we must solve (13) where $g$ is the Riemannian metric on $\mathbf{S}_{R}^{d}$ (e.g., (10)) and $\delta_{g}$ is the Dirac delta distribution on the manifold $\mathbf{S}_{R}^{d}$ (e.g., (6)). In general since we can add any harmonic function to an opposite antipodal fundamental solution of Laplace's equation and still have an opposite antipodal fundamental solution, we will use this freedom to make our fundamental solution as simple as possible. It is reasonable to expect that there exists a particular spherically symmetric fundamental solution $\mathcal{A}_{R}^{d}$ on the hypersphere with pure 'radial', $\theta:=d\left(\widehat{\mathbf{x}}, \widehat{\mathbf{x}}^{\prime}\right)$ (e.g., (3)), and constant angular dependence due to the influence of the point-like nature of the Dirac delta distribution in (13). For a spherically symmetric solution to the Laplace equation, the corresponding $\Delta_{\mathbf{S}^{d-1}}$ term in (11) vanishes since only the $l=0$ term survives in (15). In other words we expect there to exist an opposite antipodal fundamental solution of Laplace's equation on $\mathbf{S}_{R}^{d}$ such that $\mathcal{A}_{R}^{d}\left(\mathbf{x}, \mathbf{x}^{\prime}\right)=f(\theta)$ (cf. (3)), where $R$ is a parameter of this fundamental solution.

We will prove that on the $R$-radius hypersphere $\mathbf{S}_{R}^{d}$, an opposite antipodal Green's function for the Laplace operator (fundamental solution of Laplace's equation) can be given as follows.

Theorem 1. Let $d \in\{2,3, \ldots\}$. Define $\mathcal{I}_{d}:(0, \pi) \rightarrow \mathbf{R}$ as

$$
\mathcal{I}_{d}(\theta):=\int_{\theta}^{\pi / 2} \frac{\mathrm{d} x}{\sin ^{d-1} x},
$$

$\mathbf{x}, \mathbf{x}^{\prime} \in \mathbf{S}_{R}^{d}$, and $\mathcal{A}_{R}^{d}:\left(\mathbf{S}_{R}^{d} \times \mathbf{S}_{R}^{d}\right) \backslash\left\{(\mathbf{x}, \mathbf{x}): \mathbf{x} \in \mathbf{S}_{R}^{d}\right\} \rightarrow \mathbf{R}$ defined such that

$$
\mathcal{A}_{R}^{d}\left(\mathbf{x}, \mathbf{x}^{\prime}\right):=\frac{\Gamma(d / 2)}{2 \pi^{d / 2} R^{d-2}} \mathcal{I}_{d}(\theta)
$$

where $\theta:=\cos ^{-1}\left(\left[\widehat{\mathbf{x}}, \widehat{\mathbf{x}}^{\prime}\right]\right)$ is the geodesic distance between $\widehat{\mathbf{x}}$ and $\widehat{\mathbf{x}}^{\prime}$ on the unit radius hypersphere $\mathbf{S}^{d}$, with $\widehat{\mathbf{x}}=\mathbf{x} / R, \widehat{\mathbf{x}}^{\prime}=\mathbf{x}^{\prime} / R$, then $\mathcal{A}_{R}^{d}$ is an opposite antipodal fundamental solution for $-\Delta$ where $\Delta$ is the Laplace-Beltrami operator on $\mathbf{S}_{R}^{d}$. Moreover,

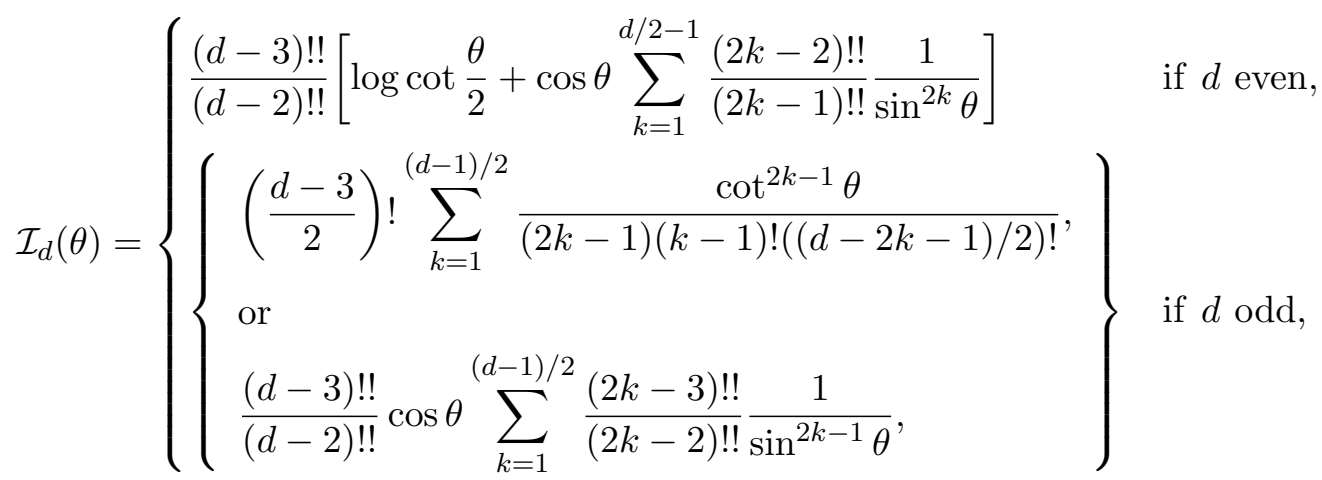

$$
\begin{aligned}
& =\left\{\begin{array}{l}
\cos \theta{ }_{2} F_{1}\left(\frac{1}{2}, \frac{d}{2} ; \frac{3}{2} ; \cos ^{2} \theta\right), \\
\frac{\cos \theta}{\sin ^{d-2} \theta}{ }_{2} F_{1}\left(1, \frac{3-d}{2} ; \frac{3}{2} ; \cos ^{2} \theta\right), \\
\frac{(d-2) !}{\Gamma(d / 2) 2^{d / 2-1}} \frac{1}{(\sin \theta)^{d / 2-1}} Q_{d / 2-1}^{1-d / 2}(\cos \theta) .
\end{array}\right.
\end{aligned}
$$

Proof. Since a spherically symmetric choice for an opposite antipodal fundamental solution satisfies Laplace's equation everywhere except at $\theta \in\{0, \pi\}$, we may first set $g=f^{\prime}$ in (11) and solve the first-order ordinary differential equation

$$
g^{\prime}+(d-1) \cos \theta g=0,
$$


which is integrable and clearly has the general solution

$$
g(\theta)=\frac{\mathrm{d} f}{\mathrm{~d} \theta}=c_{0}(\sin \theta)^{1-d},
$$

where $c_{0} \in \mathbf{R}$ is a constant. Now we integrate (19) to obtain an opposite antipodal fundamental solution for the Laplacian on $\mathbf{S}_{R}^{d}$

$$
\mathcal{A}_{R}^{d}\left(\mathbf{x}, \mathbf{x}^{\prime}\right)=c_{0} \mathcal{I}_{d}(\theta)+c_{1},
$$

where $\mathcal{I}_{d}:(0, \pi) \rightarrow \mathbf{R}$ is defined as

$$
\mathcal{I}_{d}(\theta):=\int_{\theta}^{\pi / 2} \frac{\mathrm{d} x}{\sin ^{d-1} x},
$$

and $c_{0}, c_{1} \in \mathbf{R}$ are constants (independent of $\theta$ ) which depend on $d$ and $R$. Notice that we can add any harmonic function to (20) and still have an opposite antipodal fundamental solution of the Laplacian since an opposite antipodal fundamental solution of the Laplacian must satisfy

$$
\int_{\mathbf{S}_{R}^{d}}(-\Delta \varphi)\left(\mathbf{x}^{\prime}\right) \mathcal{A}_{R}^{d}\left(\mathbf{x}, \mathbf{x}^{\prime}\right) \operatorname{dvol}_{g}^{\prime}=\varphi(\mathbf{x}),
$$

for all $\varphi \in \mathcal{S}\left(\mathbf{S}_{R}^{d}\right)$, where $\mathcal{S}$ is the space of test functions, and $d \mathrm{vol}_{g}^{\prime}$ is the Riemannian (volume) measure on $\mathbf{S}_{R}^{d}$ in the primed coordinates. Notice that our fundamental solution of Laplace's equation on the hypersphere (20), has the property that it tends towards $+\infty$ as $\theta \rightarrow 0^{+}$and tends towards $-\infty$ as $\theta \rightarrow \pi^{-}$. Therefore our fundamental solution attains all real values. As an aside, by the definition therein (see $[16,17]), \mathbf{S}_{R}^{d}$ is a parabolic manifold. Since the hypersphere $\mathbf{S}_{R}^{d}$ is bi-hemispheric, we expect that an opposite antipodal fundamental solution of Laplace's equation on the hypersphere should vanish at $\theta=\pi / 2$. It is therefore convenient to set $c_{1}=0$ leaving us with

$$
\mathcal{A}_{R}^{d}\left(\mathbf{x}, \mathbf{x}^{\prime}\right)=c_{0} \mathcal{I}_{d}(\theta) .
$$

In Euclidean space $\mathbf{R}^{d}$, a Green's function for Laplace's equation (fundamental solution for the Laplacian) is well-known and is given by the following expression (see [13, p. 94], [14, p. 17], [3, p. 211], [7, p. 6]). Let $d \in \mathbf{N}$. Define

$$
\mathcal{G}^{d}\left(\mathbf{x}, \mathbf{x}^{\prime}\right)= \begin{cases}\frac{\Gamma(d / 2)}{2 \pi^{d / 2}(d-2)}\left\|\mathbf{x}-\mathbf{x}^{\prime}\right\|^{2-d} & \text { if } d=1 \text { or } d \geq 3 \\ \frac{1}{2 \pi} \log \left\|\mathbf{x}-\mathbf{x}^{\prime}\right\|^{-1} & \text { if } d=2\end{cases}
$$

then $\mathcal{G}^{d}$ is a fundamental solution for $-\Delta$ in Euclidean space $\mathbf{R}^{d}$, where $-\Delta$ is the positive Laplace operator on $\mathbf{R}^{d}$. Note that most authors only present the above theorem for the case $d \geq 2$ but it is easily-verified to also be valid for the case $d=1$ as well.

The hypersphere $\mathbf{S}_{R}^{d}$, being a manifold, must behave locally like Euclidean space $\mathbf{R}^{d}$. Therefore for small $\theta$ we have $\mathrm{e}^{\theta} \simeq 1+\theta$ and $\mathrm{e}^{-\theta} \simeq 1-\theta$ and in that limiting regime

$$
\mathcal{I}_{d}(\theta) \approx \int_{\theta}^{1} \frac{\mathrm{d} x}{x^{d-1}} \simeq \begin{cases}-\log \theta & \text { if } d=2, \\ \frac{1}{\theta^{d-2}} & \text { if } d \geq 3,\end{cases}
$$

which has exactly the same singularity as a Euclidean fundamental solution. Therefore the proportionality constant $c_{0}$ is obtained by matching locally to a Euclidean fundamental solution

$$
\mathcal{A}_{R}^{d}=c_{0} \mathcal{I}_{d} \simeq \mathcal{G}^{d}
$$

in a small neighborhood of the singularity at $\mathbf{x}=\mathbf{x}^{\prime}$, as the curvature vanishes, i.e., $R \rightarrow \infty$. 
We have shown how to compute an opposite antipodal fundamental solution of the LaplaceBeltrami operator on the hypersphere in terms of an improper integral (21). We now prove several equivalent finite summation expressions for $\mathcal{I}_{d}(\theta)$. We wish to compute the antiderivative $\mathfrak{I}_{m}:(0, \pi) \rightarrow \mathbf{R}$, which is defined to within a constant in $x$ as

$$
\mathfrak{I}_{m}(x):=\int \frac{\mathrm{d} x}{\sin ^{m} x},
$$

where $m \in \mathbf{N}$. This antiderivative satisfies the following recurrence relation

$$
\mathfrak{I}_{m}(x)=-\frac{\cos x}{(m-1) \sin ^{m-1} x}+\frac{(m-2)}{(m-1)} \mathfrak{I}_{m-2}(x),
$$

which follows from the identity

$$
\frac{1}{\sin ^{m} x}=\frac{1}{\sin ^{m-2} x}+\frac{\cos x}{\sin ^{m} x} \cos x,
$$

and integration by parts. The antiderivative $\mathfrak{I}_{m}(x)$ naturally breaks into two separate classes, namely

$$
\int \frac{\mathrm{d} x}{\sin ^{2 n+1} x}=-\frac{(2 n-1) ! !}{(2 n) ! !}\left[\log \cot \frac{x}{2}+\cos x \sum_{k=1}^{n} \frac{(2 k-2) ! !}{(2 k-1) ! !} \frac{1}{\sin ^{2 k} x}\right]+C,
$$

and

$$
\int \frac{\mathrm{d} x}{\sin ^{2 n} x}=\left\{\begin{array}{l}
-\frac{(2 n-2) ! !}{(2 n-1) ! !} \cos x \sum_{k=1}^{n} \frac{(2 k-3) ! !}{(2 k-2) ! !} \frac{1}{\sin ^{2 k-1} x}+C, \quad \text { or } \\
-(n-1) ! \sum_{k=1}^{n} \frac{\cot ^{2 k-1} x}{(2 k-1)(k-1) !(n-k) !}+C
\end{array}\right.
$$

where $C$ is a constant. The double factorial $(\cdot) ! !:\{-1,0,1, \ldots\} \rightarrow \mathbf{N}$ is defined by

$$
n ! !:= \begin{cases}n \cdot(n-2) \cdots 2 & \text { if } n \text { even } \geq 2 \\ n \cdot(n-2) \cdots 1 & \text { if } n \text { odd } \geq 1 \\ 1 & \text { if } n \in\{-1,0\} .\end{cases}
$$

Note that $(2 n) ! !=2^{n} n$ ! for $n \in \mathbf{N}_{0}$. The finite summation formulae for $\mathfrak{I}_{m}(x)$ all follow trivially by induction using (25) and the binomial expansion (cf. [28, equation (1.2.2)])

$$
\left(1+\cos ^{2} x\right)^{n}=n ! \sum_{k=0}^{n} \frac{\cot ^{2 k} x}{k !(n-k) !} .
$$

The formulae (26) and (27) are essentially equivalent to [15, equations (2.515.1) and (2.515.2)], except [15, equation (2.515.2)] is in error with the factor $28^{k}$ being replaced with $2^{k}$. This is also verified in the original citing reference [36]. By applying the limits of integration from the definition of $\mathcal{I}_{d}(\theta)$ in (21) to (26) and (27) we obtain the following finite summation expressions

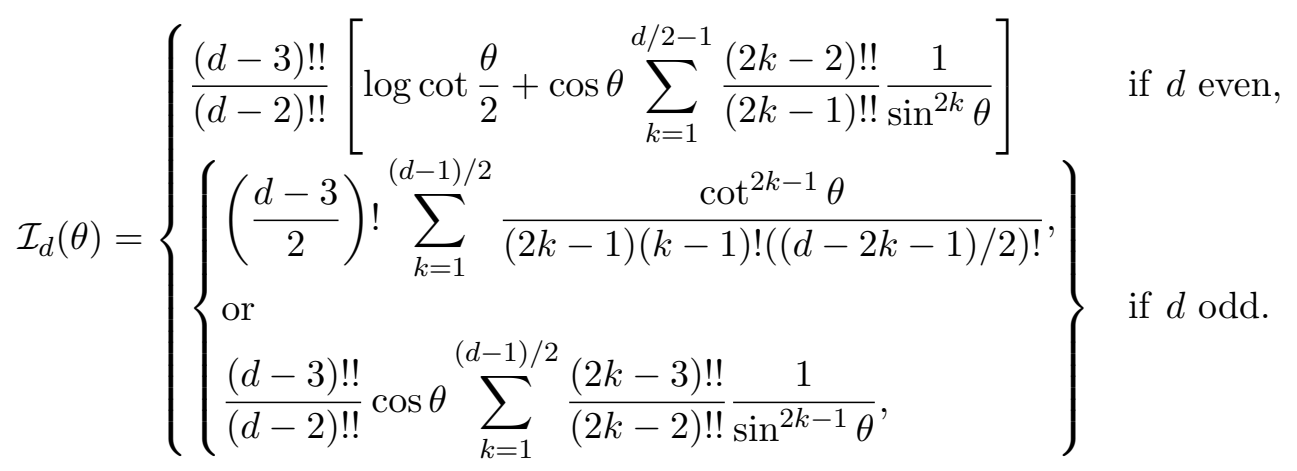


Moreover, the antiderivative (indefinite integral) can be given in terms of the Gauss hypergeometric function as

$$
\int \frac{\mathrm{d} \theta}{\sin ^{d-1} \theta}=-\cos \theta_{2} F_{1}\left(\frac{1}{2}, \frac{d}{2} ; \frac{3}{2} ; \cos ^{2} \theta\right)+C,
$$

where $C \in \mathbf{R}$. This is verified as follows. By using

$$
\frac{d}{d z}{ }_{2} F_{1}(a, b ; c ; z)=\frac{a b}{c}{ }_{2} F_{1}(a+1, b+1 ; c+1 ; z)
$$

(see [28, equation (15.5.1)]), and the chain rule, we can show that

$$
\begin{aligned}
& -\frac{\mathrm{d}}{\mathrm{d} \theta} \cos \theta_{2} F_{1}\left(\frac{1}{2}, \frac{d}{2} ; \frac{3}{2} ; \cos ^{2} \theta\right) \\
& \quad=\sin \theta\left[{ }_{2} F_{1}\left(\frac{1}{2}, \frac{d}{2} ; \frac{3}{2} ; \cos ^{2} \theta\right)+\frac{d}{3} \cos ^{2} \theta_{2} F_{1}\left(\frac{3}{2}, \frac{d+2}{2} ; \frac{5}{2} ; \cos ^{2} \theta\right)\right] .
\end{aligned}
$$

The second hypergeometric function can be simplified using Gauss' relations for contiguous hypergeometric functions, namely

$$
z_{2} F_{1}(a+1, b+1 ; c+1 ; z)=\frac{c}{a-b}\left[{ }_{2} F_{1}(a, b+1 ; c ; z)-{ }_{2} F_{1}(a+1, b ; c ; z)\right]
$$

(see $[8$, p. 58]), and

$$
{ }_{2} F_{1}(a, b+1 ; c ; z)=\frac{b-a}{b}{ }_{2} F_{1}(a, b ; c ; z)+\frac{a}{b}{ }_{2} F_{1}(a+1, b ; c ; z)
$$

(see [28, equation (15.5.12)]). By applying these formulae, the term with the hypergeometric function cancels leaving only a term which is proportional to a binomial through

$$
{ }_{2} F_{1}(a, b ; b ; z)=(1-z)^{-a}
$$

(see [28, equation (15.4.6)]), which reduces to $1 / \sin ^{d-1} \theta$. By applying the limits of integration from the definition of $\mathcal{I}_{d}(\theta)$ in (21) to (29), we obtain the following Gauss hypergeometric representation

$$
\mathcal{I}_{d}(\theta)=\cos \theta_{2} F_{1}\left(\frac{1}{2}, \frac{d}{2} ; \frac{3}{2} ; \cos ^{2} \theta\right) .
$$

Using (30), we can write another expression for $\mathcal{I}_{d}(\theta)$. Applying Euler's transformation

$$
{ }_{2} F_{1}(a, b ; c ; z)=(1-z)^{c-a-b}{ }_{2} F_{1}(c-a, c-b ; c ; z)
$$

(see $[1$, equation $(2.2 .7)])$, to $(30)$ produces

$$
\mathcal{I}_{d}(\theta)=\frac{\cos \theta}{\sin ^{d-2} \theta}{ }_{2} F_{1}\left(1, \frac{3-d}{2} ; \frac{3}{2} ; \cos ^{2} \theta\right) .
$$

Our derivation for an opposite antipodal fundamental solution of Laplace's equation on the $R$-radius hypersphere in terms of Ferrers functions of the second kind is as follows. If we let $\nu+\mu=0$ in the definition of the Ferrers function of the second kind $\mathrm{Q}_{\nu}^{\mu}:(-1,1) \rightarrow \mathbf{C}(17)$, we derive

$$
\mathrm{Q}_{\nu}^{-\nu}(x)=\frac{\sqrt{\pi}}{2^{\nu}} \frac{x\left(1-x^{2}\right)^{\nu / 2}}{\Gamma\left(\nu+\frac{1}{2}\right)}{ }_{2} F_{1}\left(\frac{1}{2}, \nu+1 ; \frac{3}{2} ; x^{2}\right),
$$


for all $\nu \in \mathbf{C}$. If we let $\nu=d / 2-1$ and substitute $x=\cos \theta$, then we have

$$
\mathrm{Q}_{d / 2-1}^{1-d / 2}(\cos \theta)=\frac{\sqrt{\pi}}{2^{d / 2-1}} \frac{\cos \theta \sin ^{d / 2-1} \theta}{\Gamma\left(\frac{d-1}{2}\right)}{ }_{2} F_{1}\left(\frac{1}{2}, \frac{d}{2} ; \frac{3}{2} ; \cos ^{2} \theta\right) .
$$

Using the duplication formula for gamma functions (18), then through (31) we have

$$
\mathcal{I}_{d}(\theta)=\frac{(d-2) !}{\Gamma(d / 2) 2^{d / 2-1}} \frac{1}{\sin ^{d / 2-1} \theta} \mathrm{Q}_{d / 2-1}^{1-d / 2}(\cos \theta) .
$$

We have therefore verified that the harmonics computed in Section 3.1, namely $u_{2,+}^{d, 0}(\cos \theta)(16)$, give an alternate form for an opposite antipodal fundamental solution of the Laplacian on the hypersphere.

The constant $c_{0}$ in an opposite antipodal fundamental solution for the Laplace operator on the hypersphere $\mathbf{S}_{R}^{d}(22)$ is computed by locally matching up, through (24), to the singularity of an opposite antipodal fundamental solution for the Laplace operator in Euclidean space (23). The coefficient $c_{0}$ depends on $d$ and $R$. For $d \geq 3$ we take the asymptotic expansion for $c_{0} \mathcal{I}_{d}(\theta)$ as $\theta \rightarrow 0^{+}$, and match this to a fundamental solution of Laplace's equation for Euclidean space (23). This yields

$$
c_{0}=\frac{\Gamma(d / 2)}{2 \pi^{d / 2}} .
$$

For $d=2$ we take the asymptotic expansion for

$$
c_{0} \mathcal{I}_{2}(\theta)=-c_{0} \log \tan \frac{\theta}{2} \simeq c_{0} \log \left\|\mathbf{x}-\mathbf{x}^{\prime}\right\|^{-1},
$$

as $\theta \rightarrow 0^{+}$, and match this to $\mathcal{G}^{2}\left(\mathbf{x}, \mathbf{x}^{\prime}\right)=(2 \pi)^{-1} \log \left\|\mathbf{x}-\mathbf{x}^{\prime}\right\|^{-1}$, therefore $c_{0}=(2 \pi)^{-1}$. This exactly matches (33) for $d=2$. The $R$ dependence of $c_{0}$ originates from (21), where $x$ and $\theta$ represents geodesic distances (cf. (3)). The distance $r \in[0, \infty)$ along a geodesic, as measured from the origin of $\mathbf{S}_{R}^{d}$, is given by $r=\theta R$. To show that an opposite antipodal fundamental solution (22) reduces to the Euclidean fundamental solution at small distances (see for instance [23]), we examine the flat-space limit of zero curvature. In order to do this, we take the limit $\theta \rightarrow 0^{+}$ and $R \rightarrow \infty$ of (21) with the substitution $x=r / R$ which produces a factor of $R^{d-2}$. So an opposite antipodal fundamental solution of Laplace's equation on the Riemannian manifold $\mathbf{S}_{R}^{d}$ is given by

$$
\mathcal{A}_{R}^{d}\left(\mathbf{x}, \mathbf{x}^{\prime}\right):=\frac{\Gamma(d / 2)}{2 \pi^{d / 2} R^{d-2}} \mathcal{I}_{d}(\theta) .
$$

This completes the proof.

Apart from the well-known historical results in two and three dimensions, the closed form expressions for an opposite antipodal fundamental solution of Laplace's equation on the $R$-radius hypersphere given by Theorem 1 in Section 3.2 appear to be new. Furthermore, the Ferrers function representations in Section 3.1 for the radial harmonics on the $R$-radius hypersphere do not appear to be have previously appeared in the literature.

\subsection{Some examples for $\mathcal{I}_{d}(\theta)$ and $\mathbf{Q}_{d / 2-1}^{1-d / 2}(\cos \theta)$}

We would now like to express the integral $\mathcal{I}_{d}(\cos \theta)$ for $d \in\{2, \ldots, 7\}$ in terms of trigonometric and/or logarithmic functions. The integral $\mathcal{I}_{d}$ can be computed using elementary methods through its definition (21). In $d=2$ we have

$$
\mathcal{I}_{2}(\theta)=\int_{\theta}^{\pi / 2} \frac{\mathrm{d} x}{\sin x}=\frac{1}{2} \log \frac{\cos \theta+1}{\cos \theta-1}=\log \cot \frac{\theta}{2},
$$


and in $d=3$ we have

$$
\mathcal{I}_{3}(\theta)=\int_{\theta}^{\pi / 2} \frac{\mathrm{d} x}{\sin ^{2} x}=\cot \theta
$$

In $d \in\{4,5,6,7\}$ we have

$$
\begin{aligned}
& \mathcal{I}_{4}(\theta)=\frac{1}{2} \log \cot \frac{\theta}{2}+\frac{\cos \theta}{2 \sin ^{2} \theta}, \\
& \mathcal{I}_{5}(\theta)=\cot \theta+\frac{1}{3} \cot ^{3} \theta, \\
& \mathcal{I}_{6}(\theta)=\frac{3}{8} \log \cot \frac{\theta}{2}+\frac{3 \cos \theta}{8 \sin ^{2} \theta}+\frac{\cos \theta}{4 \sin ^{2} \theta}, \quad \text { and } \\
& \mathcal{I}_{7}(\theta)=\cot \theta+\frac{2}{3} \cot ^{3} \theta+\frac{1}{5} \cot ^{5} \theta .
\end{aligned}
$$

Equivalently, some relevant Ferrers functions of the second kind $\mathrm{Q}_{d / 2-1}^{1-d / 2}(\cos \theta)$ for $d \in\{2, \ldots, 7\}$ are (cf. (28) and (32))

$$
\begin{aligned}
& \mathrm{Q}_{0}(\cos \theta)=\log \cot \frac{\theta}{2}, \\
& \frac{1}{(\sin \theta)^{1 / 2}} \mathrm{Q}_{1 / 2}^{-1 / 2}(\cos \theta)=\sqrt{\frac{\pi}{2}} \cot \theta, \\
& \frac{1}{\sin \theta} \mathrm{Q}_{1}^{-1}(\cos \theta)=\frac{1}{2} \log \cot \frac{\theta}{2}+\frac{\cos \theta}{2 \sin ^{2} \theta}, \\
& \frac{1}{(\sin \theta)^{3 / 2}} \mathrm{Q}_{3 / 2}^{-3 / 2}(\cos \theta)=\frac{1}{2} \sqrt{\frac{\pi}{2}}\left(\cot \theta+\frac{1}{3} \cot ^{3} \theta\right), \\
& \frac{1}{(\sin \theta)^{2}} \mathrm{Q}_{2}^{-2}(\cos \theta)=\frac{1}{8} \log \cot \frac{\theta}{2}+\frac{\cos \theta}{8 \sin ^{2} \theta}+\frac{\cos \theta}{12 \sin ^{4} \theta}, \quad \text { and } \\
& \frac{1}{(\sin \theta)^{5 / 2}} \mathrm{Q}_{5 / 2}^{-5 / 2}(\cos \theta)=\frac{1}{8} \sqrt{\frac{\pi}{2}}\left(\cot \theta+\frac{2}{3} \cot ^{3} \theta+\frac{1}{5} \cot ^{5} \theta\right) .
\end{aligned}
$$

\section{Acknowledgments}

Much thanks to Ernie Kalnins, Willard Miller Jr., George Pogosyan, and Charles Clark for valuable discussions. Much thanks as well to Richard Chapling for his comments in [4], in reference to an original version of the present paper and its implications. I would like to express my sincere gratitude to the anonymous referees and an editor at SIGMA whose helpful comments improved this paper. This work was partly conducted while H.S. Cohl was a National Research Council Research Postdoctoral Associate in the Information Technology Laboratory at the National Institute of Standards and Technology, Gaithersburg, Maryland, USA.

\section{References}

[1] Andrews G.E., Askey R., Roy R., Special functions, Encyclopedia of Mathematics and its Applications, Vol. 71, Cambridge University Press, Cambridge, 1999.

[2] Berakdar J., Concepts of highly excited electronic systems, Wiley-VCH, New York, 2003.

[3] Bers L., John F., Schechter M. (Editors), Partial differential equations, Interscience Publishers, New York, 1964.

[4] Chapling R., A hypergeometric integral with applications to the fundamental solution of Laplace's equation on hyperspheres, SIGMA 12 (2016), 079, 20 pages, arXiv:1508.06689. 
[5] Cooper J.W., Fano U., Prats F., Classification of two-electron excitation levels of helium, Phys. Rev. Lett. 10 (1963), 518-521.

[6] Delves L.M., Tertiary and general-order collisions. II, Nuclear Phys. 20 (1960), 275-308.

[7] Doob J.L., Classical potential theory and its probabilistic counterpart, Grundlehren der Mathematischen Wissenschaften, Vol. 262, Springer-Verlag, New York, 1984.

[8] Erdélyi A., Magnus W., Oberhettinger F., Tricomi F.G., Higher transcendental functions, Vol. I, Robert E. Krieger Publishing Co. Inc., Melbourne, Fla., 1981.

[9] Erdélyi A., Magnus W., Oberhettinger F., Tricomi F.G., Higher transcendental functions, Vol. II, Robert E. Krieger Publishing Co. Inc., Melbourne, Fla., 1981.

[10] Fano U., Correlations of two excited electrons, Rep. Progr. Phys. 46 (1983), 97-165.

[11] Fock V., On the Schrödinger equation of the helium atom. I, Norske Vid. Selsk. Forhdl. 31 (1958), no. 22, 7 pages.

[12] Fock V., On the Schrödinger equation of the helium atom. II, Norske Vid. Selsk. Forhdl. 31 (1958), no. 23, 8 pages.

[13] Folland G.B., Introduction to partial differential equations, Mathematical Notes, Princeton University Press, Princeton, N.J., 1976.

[14] Gilbarg D., Trudinger N.S., Elliptic partial differential equations of second order, 2nd ed., Grundlehren der mathematischen Wissenschaften, Vol. 224, Springer-Verlag, Berlin, 1983.

[15] Gradshteyn I.S., Ryzhik I.M., Table of integrals, series, and products, 7th ed., Elsevier/Academic Press, Amsterdam, 2007.

[16] Grigor'yan A.A., Existence of the Green's function on a manifold, Russ. Math. Surv. 38 (1983), no. 1, 190-191.

[17] Grigor'yan A.A., On the existence of positive fundamental solutions of the Laplace equation on Riemannian manifolds, Math. USSR Sb. 56 (1987), 349-358.

[18] Grigor'yan A.A., Heat kernel and analysis on manifolds, AMS/IP Studies in Advanced Mathematics, Vol. 47, Amer. Math. Soc., Providence, RI, International Press, Boston, MA, 2009.

[19] Higgs P.W., Dynamical symmetries in a spherical geometry. I, J. Phys. A: Math. Gen. 12 (1979), 309-323.

[20] Izmest'ev A.A., Pogosyan G.S., Sissakian A.N., Winternitz P., Contractions of Lie algebras and separation of variables. The $n$-dimensional sphere, J. Math. Phys. 40 (1999), 1549-1573.

[21] Izmest'ev A.A., Pogosyan G.S., Sissakian A.N., Winternitz P., Contractions of Lie algebras and the separation of variables: interbase expansions, J. Phys. A: Math. Gen. 34 (2001), 521-554.

[22] Izmest'ev A.A., Pogosyan G.S., Sissakian A.N., Winternitz P., Contraction and interbases expansions on $n$-sphere, in Quantum Theory and Symmetries (Kraków, 2001), World Sci. Publ., River Edge, NJ, 2002, 389-395.

[23] Kalnins E.G., Miller W. Jr., Pogosyan G.S., The Coulomb-oscillator relation on $n$-dimensional spheres and hyperboloids, Phys. Atomic Nuclei 65 (2002), 1086-1094.

[24] Lee J.M., Riemannian manifolds, Graduate Texts in Mathematics, Vol. 176, Springer-Verlag, New York, 1997.

[25] Leemon H.I., Dynamical symmetries in a spherical geometry. II, J. Phys. A: Math. Gen. 12 (1979), 489-501.

[26] Lin C.D., Hyperspherical coordinate approach to atomic and other Coulombic three-body systems, Phys. Rep. 257 (1995), 1-83.

[27] Olevskiŭ M.N., Triorthogonal systems in spaces of constant curvature in which the equation $\Delta_{2} u+\lambda u=0$ allows a complete separation of variables, Mat. Sbornik N.S. 27 (1950), 379-426.

[28] Olver F.W.J., Olde Daalhuis A.B., Lozier D.W., Schneider B.I., Boisvert R.F., Clark C.W., Miller B.R., Saunders B.V. (Editors), NIST digital library of mathematical functions, Release 1.0.21 of 2018-12-15, available at http://dlmf.nist.gov.

[29] Oprea J., Differential geometry and its applications, 2nd ed., Classroom Resource Materials Series, Mathematical Association of America, Washington, DC, 2007.

[30] Pack R.T., Parker G.A., Quantum reactive scattering in 3 dimensions using hyperspherical (APH) coordinates. Theory, J. Chem. Phys. 87 (1987), 3888-3921.

[31] Schrödinger E., Eigenschwingungen des sphärischen Raumes, Comment. Pontificia Acad. Sci. 2 (1938), 321-364. 
[32] Schrödinger E., A method of determining quantum-mechanical eigenvalues and eigenfunctions, Proc. Roy. Irish Acad. Sect. A 46 (1940), 9-16.

[33] Smith F.T., Generalized angular momentum in many-body collisions, Phys. Rev. 120 (1960), $1058-1069$.

[34] Takeuchi M., Modern spherical functions, Translations of Mathematical Monographs, Vol. 135, Amer. Math. Soc., Providence, RI, 1994.

[35] Thurston W.P., Three-dimensional geometry and topology, Vol. 1, Princeton Mathematical Series, Vol. 35, Princeton University Press, Princeton, NJ, 1997.

[36] Timofeev A.F., Integration of functions, OGIZ, Moscow - Leningrad, 1948 (in Russian).

[37] Vilenkin N.Ja., Special functions and the theory of group representations, Translations of Mathematical Monographs, Vol. 22, Amer. Math. Soc., Providence, R.I., 1968.

[38] Vinitskiü S.I., Mardoyan L.G., Pogosyan G.S., Sissakian A.N., Strizh T.A., Hydrogen atom in curved space. Expansion in free solutions on a three-dimensional sphere, Phys. Atomic Nuclei 56 (1993), 321-327.

[39] Zernike F., Brinkman H.C., Hypersphärische Funktionen und die in sphärische Bereichen orthogonalen Polynome, Proc. Akad. Wet. Amsterdam 38 (1935), 161-170.

[40] Zhukov M.V., Danilin B.V., Fedorov D.V., Bang J.M., Thompson I.J., Vaagen J.S., Bound state properties of Borromean halo nuclei: ${ }^{6} \mathrm{He}$ and ${ }^{11} \mathrm{Li}$, Phys. Rep. 231 (1993), 151-199. 